Bull. Korean Math. Soc. 50 (2013), No. 6, pp. 2103-2114

http://dx.doi.org/10.4134/BKMS.2013.50.6.2103

\title{
ON THE ARCHIMEDEAN CHARACTERIZATION OF PARABOLAS
}

\author{
Dong-Soo Kim and Young Ho Kim
}

\begin{abstract}
Archimedes knew that the area between a parabola and any chord $A B$ on the parabola is four thirds of the area of triangle $\triangle A B P$ where $\mathrm{P}$ is the point on the parabola at which the tangent is parallel to $A B$. We consider whether this property (and similar ones) characterizes parabolas. We present five conditions which are necessary and sufficient for a strictly convex curve in the plane to be a parabola.
\end{abstract}

\section{Introduction}

A parabola is the set of points in the plane which are equidistant from a point $F$ called the focus and a line $l$ called the directrix. Archimedes found some interesting area properties of parabolas.

Consider the region bounded by a parabola and a chord $A B$. Let $P$ be the point on the parabola where the tangent is parallel to the chord $A B$. The line through $P$ parallel to the axis of the parabola meets chord $A B$ at a point $V$. Then, he showed that the area of the parabolic region is $a|P V|^{3 / 2}$ for some constant $a$, which depends only on the parabola.

Furthermore, he proved that the area of the parabolic region is $4 / 3$ times the area of triangle $\triangle A B P$ whose base is the chord and whose third vertex is $P$. For the proofs of Archimedes, see Chapter 7 of [8].

In this paper, we consider whether this property (and similar ones) characterizes parabolas. As a result, we present five conditions which are necessary and sufficient for a strictly convex curve in the plane to be a parabola.

Usually, a curve $X$ in the plane $\mathbb{R}^{2}$ is called convex if it bounds a convex domain in the plane $\mathbb{R}^{2}$.

Received January 24, 2013.

2010 Mathematics Subject Classification. 53A04.

Key words and phrases. Archimedes, area, parabola, strictly convex curve, curvature.

The first author was supported by Basic Science Research Program through the National Research Foundation of Korea (NRF) funded by the Ministry of Education, Science and Technology (2010-0022926). The second author was supported by Basic Science Research Program through the National Research Foundation of Korea (NRF) funded by the Ministry of Education, Science and Technology (2012R1A1A2042298) and supported by Kyungpook National University Research Fund, 2012. 
Hereafter, we will say that a convex curve $X$ in the plane $\mathbb{R}^{2}$ is strictly convex if the curve is smooth (that is, $C^{2}$ ) and is of positive curvature $\kappa$ with respect to the unit normal $N$ pointing to the convex side. Hence, in this case we have $\kappa(s)=\left\langle X^{\prime \prime}(s), N(X(s))\right\rangle>0$, where $X(s)$ is an arclength parametrization of $X$.

For a smooth function $f: I \rightarrow \mathbb{R}$ defined on an open interval, we will also say that $f$ is strictly convex if the graph of $f$ has positive curvature $\kappa$ with respect to the upward unit normal $N$. This condition is equivalent to the positivity of $f^{\prime \prime}(x)$ on $I$.

First of all, we prove the following characterization of parabolas:

Theorem 1. Let $X$ be the graph of a strictly convex function $f: I \rightarrow \mathbb{R}$ in the plane $\mathbb{R}^{2}$. Then $f$ is a quadratic polynomial if and only if $X$ satisfies Condition: (A) For a point $P$ on $X$ and a chord $A B$ of $X$ parallel to the tangent of $X$ at $P$, let $V$ denote the point where the line through $P$ parallel to the $y$-axis meets $A B$. Then the area of the region bounded by the curve and $A B$ is $a|P V|^{3 / 2}$, where $a$ is a positive constant which depends only on the curve $X$.

Second, we prove:

Theorem 2. Let $X$ be the graph of a strictly convex function $f: I \rightarrow \mathbb{R}$ in the plane $\mathbb{R}^{2}$. Then $f$ is a quadratic polynomial if and only if $X$ satisfies Condition: (B) For a sufficiently small $k>0$, let $X_{k}$ denote the graph of $y=f(x)+k$. For any point $V$ on $X_{k}$, let the tangent at $V$ meet the curve $X$ at $A$ and $B$. Then the region $S$ bounded by $X$ and the chord $A B$ has constant area (say, $\phi(k))$ independent of the choice of $V$.

Since $|P V|=k$, Theorem 1 is a special case of Theorem 2 for $\phi(k)=a k^{3 / 2}$, where $a$ is a constant.

Now, for an arbitrary strictly convex curve $X$ in the plane $\mathbb{R}^{2}$ which is not necessarily the graph of a function, we consider the following condition:

(C) For a point $P$ on $X$ and a chord $A B$ of $X$ parallel to the tangent of $X$ at $P$, the area of the region bounded by the curve and $A B$ is $4 / 3$ times the area of triangle $\triangle A B P$.

Then, we prove the following characterization of parabolas, which is the main theorem of this article.

Theorem 3. Let $X$ be a strictly convex curve in the plane $\mathbb{R}^{2}$. Then $X$ is a parabola if and only if it satisfies Condition (C).

In order to prove Theorems 1, 2 and 3, first of all, in Section 2 we establish a new geometric meaning of curvature $\kappa$ of a plane convex curve $X$ at a point $P \in M$ with $\kappa(P)>0$ (Lemma 6 ). For the curvature function $\kappa$ of a plane curve, we refer to [3].

As applications of Theorem 3, we may prove some generalizations of Theorems 1 and 3 as follows. 
Corollary 4. Let $X$ be a strictly convex curve in the plane $\mathbb{R}^{2}$. Then $X$ is a parabola if and only if it satisfies Condition:

(D) For a point $P$ on $X$ and a chord $A B$ of $X$ parallel to the tangent of $X$ at $P$, the area of the region bounded by the curve and $A B$ is a $(P)|\triangle A B P|^{b(P)}$, where $a(P)$ and $b(P)$ are some functions of $P$ and $|\triangle A B P|$ denotes the area of the triangle $\triangle A B P$.

Finally, for the graph $X$ of a strictly convex function $f: I \rightarrow \mathbb{R}$ in the plane $\mathbb{R}^{2}$, we consider the following Condition:

(E) For a point $P$ on $X$ and a chord $A B$ of $X$ parallel to the tangent of $X$ at $P$, let $V$ denote the point where the line through $P$ parallel to the $y$-axis meets $A B$. Then the area of the region bounded by the curve and $A B$ is $a(P)|P V|^{b(P)}$, where $a(P)$ and $b(P)$ are some functions of $P$.

Then we prove:

Corollary 5. Let $X$ be the graph of a strictly convex function $f: I \rightarrow \mathbb{R}$ in the plane $\mathbb{R}^{2}$. Then $X$ satisfies Condition $(\mathrm{E})$ if and only if $X$ is a parabola, which is given by either a quadratic polynomial $f$ or a function $f$ in (3.26) according as the function $a(P)$ is constant or not.

It follows from Corollary 5 that Theorem 1 is a corollary of Theorem 3 .

To prove Corollaries 4 and 5 , first of all, we show that $b(P)$ must be 1 in Corollary 4 (respectively, $3 / 2$ in Corollary 5 ). Then we can show that $X$ satisfies Condition (C). Hence, it follows from Theorem 3 that Corollaries 4 and 5 hold.

Among the graphs of functions, Á. Bényi et al. proved some characterizations of parabolas $([1,2])$ and B. Richmond and T. Richmond established a dozen characterizations of parabolas using elementary techniques ([7]). In their papers, parabola means the graph of a quadratic polynomial in one variable.

For an example, consider a function $f(x)=b\{(1-c x)-\sqrt{1-2 c x}\}$ in (3.26) with $b, c>0$ defined on $I=\left(-\infty, \frac{1}{2 c}\right)$. Then, the function $f$ is strictly convex and its graph $X$ satisfies Condition (C) (but neither (A) nor (B)). Note that $X$ is not the graph of a quadratic polynomial, but an open part of the parabola given in (3.27).

Throughout this article, all curves are smooth (that is, $C^{3}$ ) and connected, unless otherwise mentioned.

\section{Preliminaries and Theorems 1 and 2}

Suppose that $X$ is a strictly convex curve in the plane $\mathbb{R}^{2}$ with the unit normal $N$ pointing to the convex side. For a fixed point $P \in X$, and for a sufficiently small $h>0$, consider the line $l$ passing through $P+h N(P)$ which is parallel to the tangent of $X$ at $P$. Let's denote by $A$ and $B$ the points where the line $l$ intersects the curve $X$. 
We denote by $S_{P}(h)$ (respectively, $R_{P}(h)$ ) the area of the region bounded by the curve $X$ and chord $A B$ (respectively, of the rectangle with a side $A B$ and another one on the tangent of $X$ at $P$ with height $h>0)$. We also denote by $L_{P}(h)$ the length $|A B|$ of the chord $A B$. Then we have $R_{P}(h)=h L_{P}(h)=$ $2|\triangle A B P|$, where $|\triangle A B P|$ denotes the area of the triangle $\triangle A B P$.

We may adopt a coordinate system $(x, y)$ of $\mathbb{R}^{2}$ in such a way that $P$ is taken to be the origin $(0,0)$ and the $x$-axis is the tangent line of $X$ at $P$. Furthermore, we may assume that $X$ is locally the graph of a non-negative strictly convex function $f: \mathbb{R} \rightarrow \mathbb{R}$.

For a sufficiently small $h>0$, we have

$$
\begin{aligned}
& S_{P}(h)=\int_{f(x)<h}\{h-f(x)\} d x, \\
& R_{P}(h)=h L_{P}(h)=h \int_{f(x)<h} 1 d x .
\end{aligned}
$$

The integration is taken on the interval $I_{P}(h)=\{x \in \mathbb{R} \mid f(x)<h\}$.

On the other hand, we also have

$$
S_{P}(h)=\int_{y=0}^{h} L_{P}(y) d y .
$$

This shows that

$$
S_{P}^{\prime}(h)=L_{P}(h), \text { and thus } R_{P}(h)=h S_{P}^{\prime}(h) .
$$

First of all, we prove the following lemma, which acts a key role in this article.

Lemma 6. Suppose that $X$ is a strictly convex curve in the plane $\mathbb{R}^{2}$ with the unit normal $N$ pointing to the convex side. Then we have

$$
\lim _{h \rightarrow 0} \frac{1}{\sqrt{h}} L_{P}(h)=\frac{2 \sqrt{2}}{\sqrt{\kappa(P)}},
$$

where $\kappa(P)$ is the curvature of $X$ at $P$ with respect to the unit normal $N$.

Proof. As above, we may adopt a coordinate system $(x, y)$ of $\mathbb{R}^{2}$ in such a way that $P$ is taken to be the origin $(0,0)$ and $X$ is locally the graph of a nonnegative strictly convex function $f: \mathbb{R} \rightarrow \mathbb{R}$ with $f(0)=f^{\prime}(0)=0$. Then $N$ is the upward unit normal.

The Taylor's formula of $f(x)$ is given by

$$
f(x)=a x^{2}+f_{3}(x),
$$

where $a=f^{\prime \prime}(0) / 2$, and $f_{3}(x)$ is an $O\left(|x|^{3}\right)$ function. Since $\kappa(P)=f^{\prime \prime}(0)>0$, we see that $a$ is positive. 
Now, we let $x=\sqrt{h} \xi$. Then, together with (2.1), (2.4) gives

$$
\begin{aligned}
\frac{1}{\sqrt{h}} L_{P}(h) & =\frac{1}{\sqrt{h}} \int_{f(x)<h} 1 d x \\
& =\int_{a \xi^{2}+g_{3}(\sqrt{h} \xi)<1} 1 d \xi
\end{aligned}
$$

where $g_{3}(\sqrt{h} \xi)=f_{3}(\sqrt{h} \xi) / h$. Since $f_{3}$ is an $O\left(|x|^{3}\right)$ function, we have

$$
\left|g_{3}(\sqrt{h} \xi)\right| \leq C \sqrt{h}|\xi|^{3}
$$

where $C$ is a constant. As $h \rightarrow 0$, it follows from (2.5) and (2.6) that

$$
\begin{aligned}
\lim _{h \rightarrow 0} \frac{1}{\sqrt{h}} L_{P}(h) & =\int_{a \xi^{2}<1} 1 d \xi \\
& =\frac{2}{\sqrt{a}} .
\end{aligned}
$$

Since $\kappa(P)=2 a$, this completes the proof of Lemma 6 .

Remark. From Lemma 6, we get a new geometric meaning of curvature $\kappa(P)$ of a plane convex curve $X$ at a point $P \in X$ with $\kappa(P)>0$. That is, we obtain

$$
\kappa(P)=\lim _{h \rightarrow 0} \frac{8 h}{L_{P}(h)^{2}} .
$$

Now, we give a proof of Theorem 1.

Let $X$ be the graph of a strictly convex function $f: I \rightarrow \mathbb{R}$, where $I$ is an open interval. Then $N$ is given by the upward unit normal. For a fixed point $P=(x, f(x))$ on $X$ and a small number $h>0$, consider the line $l$ passing through the point $P+h N(P)$ which is parallel to the tangent to $X$ at $P$.

Then the hypothesis shows that $S_{P}(h)=a|P V|^{3 / 2}$ for small $h>0$, where $a$ is a constant depending only on $X$. Note that $|P V|=h \sec \theta$, where $f^{\prime}(x)=\tan \theta$ is the slope of the tangent line at $P$. Hence we have:

$$
\begin{aligned}
S_{P}(h) & =a(\sec \theta)^{3 / 2} h^{3 / 2} \\
& =a W(x)^{3 / 2} h^{3 / 2},
\end{aligned}
$$

where $W(x)=\sqrt{1+f^{\prime}(x)^{2}}$. Thus $(2.2)$ yields

$$
L_{P}(h)=\frac{3}{2} a W(x)^{3 / 2} h^{1 / 2}
$$

Therefore it follows from Lemma 6 that

$$
\kappa(P)=\frac{32}{9 a^{2} W(x)^{3}} .
$$

Since the curvature $\kappa(P)$ of $X$ at $P=(x, f(x))$ is given by

$$
\kappa(P)=\frac{f^{\prime \prime}(x)}{W(x)^{3}},
$$


we see that $f^{\prime \prime}(x)$ is a constant. Hence $f(x)$ is a quadratic polynomial. This completes the proof of the if part of Theorem 1.

By a straightforward calculation, it is trivial to prove the only if part of Theorem 1. This completes the proof of Theorem 1.

Second, we give a proof of Theorem 2 .

Let $X$ be the graph of a strictly convex function $f: I \rightarrow \mathbb{R}$, where $I$ is an open interval. Then $N$ is given by the upward unit normal. We fix a point $P(x, f(x))$ on $X$. For a sufficiently small $h>0$, consider the line $l$ passing through $P+h N(P)$ which is parallel to the tangent of $X$ at $P$. Let's denote by $A$ and $B$ the points where the line $l$ intersects the curve $X$.

Then the chord $A B$ is tangent to $X_{k}$ at $V(x, f(x)+k)$, where $k=h W$ and $W(x)=\sqrt{1+f^{\prime}(x)^{2}}$. The hypothesis shows that $S_{P}(h)=\phi(k)$. It follows from (2.2) that

$$
\begin{aligned}
& L_{P}(h)=S_{P}^{\prime}(h)=W(x) \phi^{\prime}(h W), \\
& R_{P}(h)=h L_{P}(h)=h W(x) \phi^{\prime}(h W) .
\end{aligned}
$$

Hence we have

$$
\frac{L_{P}(h)}{\sqrt{h}}=\frac{\phi^{\prime}(k)}{\sqrt{k}} W(x)^{3 / 2} .
$$

For a fixed point $P(x, f(x))$ on $X$, it follows from $k=h W(x)$ that $h \rightarrow 0$ is equivalent to $k \rightarrow 0$. Thus, Lemma 6 implies that

$$
\lim _{k \rightarrow 0} \frac{\phi^{\prime}(k)}{\sqrt{k}}=W(x)^{-3 / 2} \lim _{h \rightarrow 0} \frac{1}{\sqrt{h}} L_{P}(h)=\frac{2 \sqrt{2}}{\sqrt{\kappa(P)}} W(x)^{-3 / 2} .
$$

If we denote by $\alpha$ the limit of the left hand side of (2.14), which is independent of $P$, then we have

$$
\kappa(P)=\frac{8}{\alpha^{2} W(x)^{3}} .
$$

Similarly to the proof of Theorem 1 , we see that $f(x)$ is a quadratic polynomial. This completes the proof of the if part of Theorem 2 .

For a proof of the only if part of Theorem 2, see Example 1.2 in [6, p. 6]. This completes the proof of Theorem 2 .

\section{Main theorem}

In this section, we prove Theorem 3, which is the main theorem of this article.

Let $X$ denote a strictly convex curve in the plane $\mathbb{R}^{2}$ with the unit normal $N$ pointing to the convex side. Suppose that $X$ satisfies Condition (C). Then, for $P \in X$ and a sufficiently small $h>0$ we have

$$
S_{P}(h)=\frac{2}{3} R_{P}(h) .
$$


By differentiating (3.1) with respect to $h$, it follows from (2.2) that

$$
L_{P}(h)=2 h L_{P}^{\prime}(h) \text {. }
$$

Therefore, we get

$$
L_{P}(h)=c(P) \sqrt{h},
$$

where $c=c(P)$ is a constant depending on $P$. Furthermore, Lemma 6 implies that

$$
c(P)=\frac{2 \sqrt{2}}{\sqrt{\kappa(P)}} .
$$

In order to prove Theorem 3, first, we fix an arbitrary point $A$ on $X$.

As before, we take a coordinate system $(x, y)$ of $\mathbb{R}^{2}: A$ is taken to be the origin $(0,0)$ and $x$-axis is the tangent line of $X$ at $A$. Furthermore, we may regard $X$ to be locally the graph of a non-negative strictly convex function $f: \mathbb{R} \rightarrow \mathbb{R}$ with $f(0)=f^{\prime}(0)=0$ and $f^{\prime \prime}(0)>0$.

For any point $B(x, f(x))$ with $x \neq 0$, we denote by $P$ the point on $X$ such that the chord $A B$ is parallel to the tangent of $X$ at $P$. Then we have $P=(g(x), f(g(x)))$, for a function $g: \mathbb{R} \backslash\{0\} \rightarrow \mathbb{R}$ which satisfies $|g(x)|<|x|$ and

$$
x f^{\prime}(g(x))=f(x) .
$$

Since $g(x)$ tends to 0 as $x \rightarrow 0$, we may assume that $g(0)=0$.

We prove the following lemma, which plays a crucial role in the proof of Theorem 3.

Lemma 7. $f(x)$ and $g(x)$ satisfy

$$
\begin{gathered}
x^{3} f^{\prime \prime}(g(x))=8\{f(x) g(x)-x f(g(x))\}, \\
x f(x)=\frac{4}{3}\{f(x) g(x)-x f(g(x))\}+2 \int_{0}^{x} f(t) d t .
\end{gathered}
$$

Proof. Consider the triangle $\triangle A B C$, where $C$ denotes the point $(x, 0)$. Then we have $|A C|^{2}+|B C|^{2}=|A B|^{2}$. Note that by definition, $|A B|^{2}=L_{P}(h)^{2}$, where $h$ denotes the distance from $P$ to the chord $A B$. This shows that

$$
x^{2}+f(x)^{2}=L_{P}(h)^{2} .
$$

The distance $h$ from $P$ to the chord $A B$ is given by

$$
h=\frac{\epsilon\{f(x) g(x)-x f(g(x))\}}{\sqrt{x^{2}+f(x)^{2}}},
$$

where $\epsilon=1$ for $x>0$ and $\epsilon=-1$ for $x<0$.

Since the curvature $\kappa(P)$ of $X$ at $P$ is given by

$$
\kappa(P)=\frac{f^{\prime \prime}(g(x))}{\left(\sqrt{1+f^{\prime}(g(x))^{2}}\right)^{3}},
$$


it follows from (3.3), (3.4) and (3.5) that

$$
L_{p}(h)^{2}=\frac{8 h}{\kappa(P)}=\frac{8\left(x^{2}+f(x)^{2}\right)}{f^{\prime \prime}(g(x)) x^{3}}\{f(x) g(x)-x f(g(x))\} .
$$

Together with (3.8), this implies that (3.6) holds.

In order to prove (3.7), we consider the area of triangle $\triangle A B C$. Then we have

$$
\frac{\epsilon}{2} x f(x)=S_{P}(h)+\epsilon \int_{0}^{x} f(t) d t
$$

where $\epsilon=1$ for $x>0$ and $\epsilon=-1$ for $x<0$. By assumption, we have $S_{P}(h)=(4 / 3)|\triangle A B P|$. Hence we get

$$
S_{P}(h)=\frac{2 \epsilon}{3}\{f(x) g(x)-x f(g(x))\}
$$

Together with (3.12), this implies that (3.7) holds.

Next, with the help of Lemma 7 , we show that in a neighborhood of an arbitrary point $A \in X$, the curve $X$ is a parabola.

By differentiating (3.7) with respect to $x$, it follows from (3.5) that

$$
f(g(x))=g(x) f^{\prime}(x)-\frac{3}{4}\left\{x f^{\prime}(x)-f(x)\right\} .
$$

Differentiating (3.5) with respect to $x$, and using again (3.5), we get

$$
f^{\prime \prime}(g(x))=\frac{x f^{\prime}(x)-f(x)}{x^{2} g^{\prime}(x)} .
$$

On the other hand, together with (3.14), (3.6) shows that

$$
f^{\prime \prime}(g(x))=\frac{x f^{\prime}(x)-f(x)}{x^{3}}\{6 x-8 g(x)\} .
$$

It follows from (3.15) and (3.16) that

$$
\left\{x f^{\prime}(x)-f(x)\right\}\left\{8 g(x) g^{\prime}(x)-6 x g^{\prime}(x)+x\right\}=0 .
$$

Since $f(x)$ is strictly convex, we obtain

$$
8 g(x) g^{\prime}(x)-6 x g^{\prime}(x)+x=0 .
$$

If we let $y=g(x)$, then $(3.18)$ becomes $x d x+(8 y-6 x) d y=0$. By putting $y=v x$, we get a separable differential equation, and hence we can solve (3.18). Since $g(0)=0$, we see that $g(x)=x / 2, x / 4$ or

$$
g(x)=\frac{1}{4 c}(c x+1-\sqrt{1-2 c x}),
$$

where $c$ is a nonzero constant.

By differentiating (3.14) with respect to $x$, it follows from (3.5) that

$$
\left\{x g(x)-\frac{3}{4} x^{2}\right\} f^{\prime \prime}(x)+x g^{\prime}(x) f^{\prime}(x)-g^{\prime}(x) f(x)=0 .
$$


If $g(x)=x / 2$, then $(3.20)$ shows that

$$
x^{2} f^{\prime \prime}(x)-2 x f^{\prime}(x)+2 f(x)=0
$$

of which general solutions are given by $a x^{2}+b x$ for some $a, b \in \mathbb{R}$. Since $f(0)=f^{\prime}(0)=0$, it follows from (3.21) that $f(x)=a x^{2}$ for some positive constant $a$. Thus, in a neighborhood of $A$, the curve $X$ is a parabola.

If $g(x)=x / 4$, then (3.20) yields that

$$
2 x^{2} f^{\prime \prime}(x)-x f^{\prime}(x)+f(x)=0 .
$$

For some $a, b \in \mathbb{R}$, the general solutions of (3.22) are given by

$$
f(x)=a x+b \sqrt{|x|} .
$$

This contradicts to $f^{\prime}(0)=0$.

If $g(x)=\frac{1}{4 c}(c x+1-\sqrt{1-2 c x})$, it follows from (3.20) that

$$
(1-2 c x)\{\sqrt{1-2 c x}-(1-c x)\} f^{\prime \prime}(x)+c^{2} x f^{\prime}(x)-c^{2} f(x)=0 .
$$

The general solutions of (3.24) are given by

$$
f(x)=a x+b(1-\sqrt{1-2 c x}),
$$

where $a, b \in \mathbb{R}$. Since $f(x)$ satisfies $f(0)=f^{\prime}(0)=0$ and $f^{\prime \prime}(0)>0,(3.25)$ shows that

$$
f(x)=b\{(1-c x)-\sqrt{1-2 c x}\}
$$

where $b$ is a positive constant. Hence, in a neighborhood of $A$, the curve $X$ is given by

$$
b^{2} c^{2} x^{2}+2 b c x y+y^{2}-2 b y=0 .
$$

It follows from the classification theorem of quadratic polynomials in $x$ and $y$ that the curve defined by (3.27) is a parabola.

Summarizing the above discussions, we see that the curve $X$ is locally a parabola.

Finally, we show that the curve $X$ is a parabola as follows.

First, consider two parabolas $\Phi_{1}$ and $\Phi_{2}$ in the plane $\mathbb{R}^{2}$. For each $i=1,2$, let's denote by $\phi_{i}$ a connected open arc of the parabola $\Phi_{i}$.

Suppose that the two $\operatorname{arcs} \phi_{1}$ and $\phi_{2}$ share a common subarc $\phi$. We fix a point $A$ on the subarc $\phi$. As before, we take a coordinate system $(x, y)$ of $\mathbb{R}^{2}$ : $A$ is taken to be the origin $(0,0), x$-axis is the tangent line of $\phi$ at $A$ and $\phi$ lies in the upper half plane. Then for each $i=1,2$, the parabolic arc $\phi_{i}$ is locally the graph of $f_{i}$ which is either of the form $f_{i}(x)=a_{i} x^{2}$ with $a_{i}>0$ or of the form in (3.26) with $b=b_{i}>0, c=c_{i} \neq 0$. That is, the parabola $\Phi_{i}$ is of the form $y=a_{i} x^{2}$ with $a_{i}>0$ or of the form in (3.27) with $b=b_{i}>0, c=c_{i} \neq 0$.

Since $f_{1}$ is equal to $f_{2}$ around $x=0, f_{1}$ and $f_{2}$ have the same derivatives at the origin. Hence, we immediately see that $\Phi_{1}=\Phi_{2}$ because $f_{i}^{\prime \prime}(0)=$ $2 a_{i}, f_{i}^{\prime \prime \prime}(0)=0$ or $f_{i}^{\prime \prime}(0)=b_{i} c_{i}^{2}, f_{i}^{\prime \prime \prime}(0)=3 b_{i} c_{i}^{3}$ in each case for $i=1,2$. 
Next, let's fix a point $A$ on the curve $X$. Then an open $\operatorname{arc}$ of $X$ containing $A$ is a parabolic arc $\phi_{0}$ of a parabola $\Phi_{0}$. For an arbitrary point $B$ on the curve $X$, the compactness of the closed arc $A B$ of $X$ shows that there exist consecutive points $A=P_{0}, P_{1}, \ldots, P_{n}=B$ on $X$ and open $\operatorname{arcs} \phi_{0}, \phi_{1}, \ldots, \phi_{n}$ of $X$ such that 1 ) for each $i=0,1, \ldots, n, P_{i}$ lies on $\left.\phi_{i}, 2\right)$ each $\phi_{i}$ is a parabolic arc, 3) $\left\{\phi_{i}\right\}$ covers the closed arc $A B$ of $X$.

Since $\phi_{i}$ and $\phi_{i+1}$ share a common subarc for each $i=0,1, \ldots, n-1$, a successive use of the above argument shows that every $\phi_{i}$ is an arc of the parabola $\Phi_{0}$, and hence $B \in \Phi_{0}$. Therefore we see that $X$ is the parabola $\Phi_{0}$.

This completes the proof of the if part of Theorem 3.

For a proof of the only if part of Theorem 3, see Chapter 7 of [8], which is originally due to Archimedes. This completes the proof of Theorem 3.

\section{Corollaries and remarks}

In this section, first of all, we prove Corollaries 4 and 5 .

First, suppose that a strictly convex curve $X$ in the plane $\mathbb{R}^{2}$ satisfies Condition (D) with $b(P)=1$. Then we have

$$
S_{P}(h)=\frac{a(P)}{2} h L_{P}(h)
$$

By differentiating (4.1) with respect to $h$, it follows from (2.2) that

$$
(2-a(P)) L_{P}(h)=a(P) h L_{P}^{\prime}(h) .
$$

Solving (4.2), we get

$$
L_{P}(h)=c(P) h^{d(P)},
$$

where $c=c(P)$ is a constant depending on $P$ and $d(P)=(2-a(P)) / a(P)$.

It follows from (4.3) and Lemma 6 that $d(P)=1 / 2$, and hence, $a(P)=4 / 3$. Thus, the curve $X$ satisfies Condition (C).

Now, suppose that $X$ satisfies Condition (D) with $b(P) \neq 1$. Then we have

$$
S_{P}(h)=a(P) 2^{-b(P)}\left\{h L_{P}(h)\right\}^{b(P)},
$$

which shows that $b(P)>0$. By differentiating (4.4) with respect to $h$, it follows from (2.2) that

$$
L_{P}^{\prime}(h)+h^{-1} L_{P}(h)=c(P) h^{-b(P)} L_{P}(h)^{2-b(P)},
$$

where $c(P)=2^{b(P)} a(P)^{-1} b(P)^{-1}$. Solving the Bernoulli equation (4.5), we get

$$
\left\{h L_{P}(h)\right\}^{b(P)-1}=c(P)(b(P)-1) \ln h+d(P),
$$

where $d(P)$ is a constant depending on $P$.

In case $b(P)>1$, by letting $h \rightarrow 0$, (4.6) leads to a contradiction. In case $b(P) \in(0,1)$, multiplying the both sides of $(4.6)$ by $h^{\alpha(P)}$ with $\alpha(P)=$ $(1-b(P)) / 2>0$, and then by letting $h \rightarrow 0$, we get a contradiction. This shows that $b(P)$ must be 1 . 
Together with the above discussion on the case $b(P)=1$, Theorem 3 completes the proof of Corollary 4.

Next, we prove Corollary 5 .

Suppose that the graph $X$ of a strictly convex function $f: I \rightarrow \mathbb{R}$ in the plane $\mathbb{R}^{2}$ satisfies Condition (E). Then for a fixed point $P(x, f(x))$ on $X$ and for $h>0$, we have

$$
S_{P}(h)=a(P)|P V|^{b(P)} .
$$

Since $|P V|=h W(x)$ with $W(x)=\sqrt{1+f^{\prime}(x)^{2}}$, by differentiating (4.7) with respect to $h$, we get

$$
L_{P}(h)=a(P) b(P) W(x)^{b(P)} h^{b(P)-1} .
$$

Hence, it follows from Lemma 6 that $b(P)=3 / 2$. This shows that

$$
S_{P}(h)=a(P) W(x)^{3 / 2} h^{3 / 2} \quad \text { and } \quad L_{P}(h)=\frac{3}{2} a(P) W(x)^{3 / 2} \sqrt{h} .
$$

Thus we get

$$
\Delta A B P=\frac{1}{2} h L_{P}(h)=\frac{3}{4} a(P) W(x)^{3 / 2} h^{3 / 2}=\frac{3}{4} S_{P}(h),
$$

which shows that $X$ satisfies Condition (C). Therefore, it follows from the proof of Theorem 3 that $X$ is a parabola, which is given by either a quadratic polynomial $f$ or a function $f$ in $(3.26)$.

Conversely, if $f$ is a quadratic polynomial, Theorem 1 shows that the graph $X$ of $f$ satisfies Condition (E) with a constant $a(P)$ and $b(P)=3 / 2$. If $f$ is a function in (3.26), it is straightforward to show that the graph $X$ of $f$ satisfies Condition (E) with a nonconstant function $a(P)$ and $b(P)=3 / 2$.

This completes the proof of Corollary 5.

Together with (3.1)-(3.4) and Theorem 3, the same argument as in the proof of Corollary 5 shows:

Corollary 8. Let $X$ denote a strictly convex curve in the plane $\mathbb{R}^{2}$. Then, the following are equivalent.

1) $X$ satisfies Condition $(\mathrm{C})$.

2) $S_{P}(h)=a(P) h^{3 / 2}$, where $a(P)$ is a function of $P \in X$.

3) $S_{P}(h)=a(P) h^{b(P)}$, where $a(P)$ and $b(P)$ are some functions of $P \in X$.

4) $X$ is a parabola.

Remark 9. It follows from our proofs that Theorem 3 holds even if a strictly convex (hence, $C^{3}$ ) curve $X$ satisfies Condition (C) for sufficiently small $h>0$ at every point $P \in X$.

Finally, we give an example of a convex curve which satisfies Condition (C) for sufficiently small $h>0$ at every point $P \in X$, but it is not a parabola. Note that the example is not $C^{2}$, and hence it is not strictly convex either. 
Example 10. Consider the graph $X$ of a function $f: \mathbb{R} \rightarrow \mathbb{R}$ which is given by

$$
f(x)= \begin{cases}9 x^{2}, & \text { if } x<0, \\ \frac{9}{4} x^{2}, & \text { if } x \geq 0 .\end{cases}
$$

Then, the function $f$ is not $C^{2}$ at the origin, and hence the curve $X$ is not strictly convex. It is straightforward to show that if $P$ is the origin, then for all $h>0$ we have

$$
L_{P}(h)=\sqrt{h}, \quad \text { and } \quad S_{P}(h)=\frac{2}{3} R_{P}(h) .
$$

Hence $X$ satisfies Condition (C) at the origin for all $h>0$. If $P \in X$ is not the origin, then there exists a positive number $\varepsilon(P)$ such that for every positive number $h$ with $h<\varepsilon(P), X$ satisfies Condition $(\mathrm{C})$.

Thus, $X$ satisfies Condition (C) for sufficiently small $h>0$ at every point $P \in X$. But it is not a parabola.

Remark 11. In [4] and [5], the authors proved the higher dimensional versions of Theorems 1 and 2, respectively.

\section{References}

[1] Á. Bényi, P. Szeptycki, and F. Van Vleck, Archimedean properties of parabolas, Amer. Math. Monthly 107 (2000), no. 10, 945-949.

[2] _ A generalized Archimedean property, Real Anal. Exchange 29 (2003/04), no. 2, 881-889.

[3] M. P. do Carmo, Differential Geometry of Curves and Surfaces, Prentice-Hall, Englewood Cliffs, NJ, 1976.

[4] D.-S. Kim and Y. H. Kim, Some characterizations of spheres and elliptic paraboloids, Linear Algebra Appl. 437 (2012), no. 1, 113-120.

[5] - Some characterizations of spheres and elliptic paraboloids. II, Linear Algebra Appl. 438 (2013), no. 3, 1356-1364.

[6] K. Nomizu and T. Sasaki, Affine Differential Geometry, Geometry of affine immersions, Cambridge Tracts in Mathematics, 111, Cambridge University Press, Cambridge, 1994.

[7] B. Richmond and T. Richmond, How to recognize a parabola, Amer. Math. Monthly 116 (2009), no. 10, 910-922.

[8] S. Stein, Archimedes: What did he do besides cry Eureka?, Mathematical Association of America, Washington, DC, 1999.

DonG-Soo KIM

Department of Mathematics

Chonnam National University

KWANGJU 500-757, Korea

E-mail address: dosokim@chonnam.ac.kr

YOUNG Ho KIM

Department of Mathematics

KYUNGPOOK NATIONAL UNIVERSITY

TAEgu 702-701, Korea

E-mail address: yhkim@knu.ac.kr 